\title{
A focus on the genotoxicity of gold nanoparticles
}

\author{
Yadong Wang**,1(iD), Hongsheng Zhang ${ }^{2}$, Li Shi² ${ }^{2}$ Jie Xu², Guangcai Duan ${ }^{2}$ \& Haiyan \\ Yang*,2 \\ ${ }^{1}$ Department of Toxicology, Henan Center for Disease Control \& Prevention, Zhengzhou, 450016, PR China \\ ${ }^{2}$ Department of Epidemiology, School of Public Health, Zhengzhou University, Zhengzhou, 450001, PR China \\ *Author for correspondence: yhy@zzu.edu.cn \\ **Author for correspondence: Tel.: +86 3716808 9043; Fax: +86 3716808 9043; wangyd76@163.com
}

"The comet assay is very simple and cost efficient, and is relatively sensitive for screening the genotoxicity of Au-NPs in in vitro and in vivo test systems."

First draft submitted: 29 September 2019; Accepted for publication: 21 November 2019; Published online: 30 January 2020

Keywords: genotoxicity • gold nanoparticles $\bullet$ in vitro study • in vivo study

With the development of nanotechnology, nanoparticles (NPs), which are often defined as particles with at least one dimension ranging between 1 and $100 \mathrm{~nm}$, have been widely used in multiple sectors, such as industry, medicine and everyday life. Gold NPs (Au-NPs) are one of the most common metallic NPs used in the biomedical field for diagnosis, treatment and imaging due to their special characteristics, such as biocompatibility, controllable particle size and shape, ease of synthesis, controllable distribution, ease of surface functionalization, chemical stability, plasmonic properties and tunable optical properties. However, recent studies have focused on the adverse effects of Au-NPs on human health, especially genotoxicity, which is responsible for deleterious effects on cellular genetic material and is commonly associated with tumorigenesis. In this paper, we summarized the genotoxicity of Au-NPs based on in vitro and in vivo studies.

\section{In vitro studies}

The genotoxicity of Au-NPs was investigated in 13 in vitro experiments that included the Ames test, SOS chromotest, comet assay, micronucleus $(\mathrm{MN})$ assay, chromosome aberration (CA) assay and liquid chromatography/tandem mass spectrometry (LC/MS/MS) analysis.

\section{Comet assay}

A total of 12 studies focused on the genotoxicity of Au-NPs using a comet assay in mammalian cells. Among them, six studies reported positive results. Human peripheral blood lymphocytes (PBLs) and murine macrophage cell line Raw264.7 were exposed to various concentrations $(0.1,1,10$ and $100 \mu \mathrm{g} / \mathrm{ml})$ of 5 and $15 \mathrm{~nm} \mathrm{Au}-\mathrm{NPs}$ in a comet assay. The results showed that treatment with Au-NPs induced a significant increase in DNA strand breaks and oxidative DNA damage [1]. A concentration-dependent increase in DNA damage was observed in Chinese hamster ovary $(\mathrm{CHO})$ cells treated with Au-NPs compared with the untreated negative control cells when the $\mathrm{CHO}$ cells were exposed to $14 \mathrm{~nm}$ Au-NPs $(6.2,12.5,25$ or $50 \mu \mathrm{g} / \mathrm{ml}$ ) for $20 \mathrm{~h}$ [2]. Moreover, small airway epithelial cells were treated with $1 \mathrm{nmol} / \mathrm{l}$ of $20 \mathrm{~nm}$ Au-NPs for $72 \mathrm{~h}$, after which DNA damage was measured using a comet assay. The tail moment was significantly greater in small airway epithelial cells treated with Au-NPs compared with the negative control cells [3]. Similarly, MRC-5 human fetal lung fibroblast cells were treated with $1 \mathrm{nM}$ of $20 \mathrm{~nm}$ $\mathrm{Au}-\mathrm{NPs}$ for $72 \mathrm{~h}$ and then subjected to a comet assay. The tail moment was significantly higher in the treated group than in the untreated control group [4]. BALB/c 3T3 cells were treated with $12 \mathrm{~nm}$ Au-NPs for $4 \mathrm{~h}, 24 \mathrm{~h}, 48 \mathrm{~h}$, $15 \mathrm{~min}$ or $30 \mathrm{~min}$ and then subjected to a comet assay. The results showed an increase in DNA damage within $15 \mathrm{~min}$ and was substantial after $4 \mathrm{~h} \mathrm{[5].} \mathrm{HepG2} \mathrm{human} \mathrm{liver} \mathrm{cancer} \mathrm{cells} \mathrm{were} \mathrm{treated} \mathrm{with} 10 \mathrm{ppm} \mathrm{Au-NPs} \mathrm{(10,}$ 30 or $60 \mathrm{~nm}$ ) for $16 \mathrm{~h}$ and subjected to a comet assay. The tail moment measurement showed a significant increase 
in DNA damage in the treated group compared with the untreated control group [6]. Furthermore, HepG2 and tumoral human leukemia (HL-60) cells were exposed to different concentrations of Au-NPs (30, 50 or $90 \mathrm{~nm}$ ) for $24 \mathrm{~h}$. The Au-NP treatment induced obvious DNA damage and oxidative DNA damage according to the comet assay results [7].

Five of the 12 comet assays showed that Au-NP-induced DNA damage was dependent on the duration of exposure, particle size, type of cell line and surface coating of the NPs. Human lung adenocarcinoma A549 cells were treated with varying concentrations of Au-NPs $(3-4 \mathrm{~nm})$ for 3 or $24 \mathrm{~h}$. The results showed that the Au-NP treatment for $3 \mathrm{~h}$ did not induce obvious DNA damage, but the DNA damage was obviously increased after $24 \mathrm{~h}$ of the Au-NP treatment [8]. Thus, a series of exposure time points should be applied to evaluate the genotoxicity of Au-NPs using a comet assay. Lebedova et al. reported that 5-nm Au-NPs can significantly induce DNA damage in normal human bronchial epithelial cells as detected by a comet assay, but 50-nm Au-NPs failed to induce DNA damage [9]. Xia et al. also showed that the particle size of Au-NPs had effects on their genotoxicity. The findings from a comet assay indicated that smaller Au-NPs $(5 \mathrm{~nm})$ induced a significant increase in overall DNA damage in a dose-dependent manner in HepG2 cells, but larger Au-NPs $(20$ or $50 \mathrm{~nm}$ ) failed to induce obvious DNA damage in the same test system [10]. Together, the studies of Lebedova et al. and Xia et al. agree that smaller sized Au-NPs exhibit stronger genotoxic potential compared with larger-sized Au-NPs, which is most likely due to the ability of smaller sized Au-NPs to cross cellular membranes to reach the nucleus. Paino et al. showed that Au-NPs-citrate at $50 \mathrm{~nm}$ and Au-NP-PAMAM at 1 and $50 \mathrm{~nm}$ significantly induced increased DNA damage in HepG2 cells, as detected by a comet assay, but only Au-NPs-PAMAM at $50 \mathrm{~nm}$ induced a significant increase of DNA damage in peripheral blood mononuclear cells [11]. This finding suggests that HepG2 cells are sensitive to DNA damage induced by Au-NPs compared with peripheral blood mononuclear cells, according to the study by Paino et al., which is consistent with previous observations that different cell lines are differentially sensitive to the cytotoxic effects of Au-NPs. This may be because Au-NPs modulate physiological processes in different cell types via diverse pathways. Therefore, at least two types of cell lines should be used in the determination of the genotoxicity of $\mathrm{Au}-\mathrm{NPs}$ by a comet assay. Fraga $e$ t al. investigated the influence of surface coating on the genotoxicity of Au-NPs in HepG2 cells, as measured by a comet assay. The data showed that the exposure of HepG2 cells to Au-NPs-citrate for $24 \mathrm{~h}$, but not to MuA-Au-NPs, induced significant DNA damage compared with no exposure [12]. The effects of coating materials on the genotoxicity of Au-NPs may provide useful clues for designing Au-NPs with low toxicity and high performance for clinical and medical applications.

\section{MN assay}

Three in vitro studies investigated the genotoxicity of Au-NPs using the MN test. Positive results were reported in one of the three studies, whereas two studies had negative results. Raw264.7 and PBL cells were treated with 5 and $15 \mathrm{~nm} \mathrm{Au-NPs}$ for $48 \mathrm{~h}$. The results showed that the frequency of MN formation was significantly increased in cells exposed to Au-NPs compared with the negative control, according to a cytokinesis-block micronucleus assay [1]. CHO cells were treated with $14 \mathrm{~nm}$ Au-NPs for $20 \mathrm{~h}$. The results from the cytokinesis-block micronucleus assay showed that the Au-NP treatment did not induce a significant increase in MN formation compared with the negative control [2]. Lebedova et al. exposed human bronchial epithelial cells to Au-NPs (5 or $50 \mathrm{~nm}$ ) for $48 \mathrm{~h}$, but no clear induction of $\mathrm{MN}$ formation was observed after any of the tested doses when $\mathrm{MN}$ scoring was analyzed by flow cytometry [9]. The MN test is one of the preferred methods for determining chromosomal damage and damage to the mitotic apparatus of cells induced by conventional chemicals. The current data show that Au-NP-induced genotoxicity as measured by the MN assay depends on the cell type. Thus, two additional types of cell lines should be tested to assess the genotoxicity of Au-NPs through the MN test.

\section{CA assay}

The CA test analyzes the structural and numerical aberrations that result from failures in repair processes in cultured mammalian cells, such that breaks either rejoin or do not rejoin in abnormal configurations, so that cells undergo cell cycle arrest in the metaphase stage of mitosis. Two in vitro studies investigated the effects of Au-NPs on CA in mammalian cells, but the results were negative. When $\mathrm{CHO}$ cells were exposed to 14-nm Au-NPs for $20 \mathrm{~h}$, aberrations found in the cells treated with Au-NPs were not significantly different from those found in the negative control [2]. Xia et al. also reported no significant difference in the incidences of CA between Chinese hamster lung fibroblasts treated with Au-NPs (5, 20 or $50 \mathrm{~nm}$ ) and untreated control cells [10]. 


\section{Other in vitro studies}

Di Bucchiaico et al. showed that Au-NPs exposure induced a significant increase in centromere-positive MN formation in Raw264.7 cells and PBL cells in fluorescence in situ hybridization assay [1]. Li et al. indicated that cells treated with Au-NPs exhibited a significant increase in aberrations per cell compared with untreated control cells, according to a fluorescence in situ hybridization assay [4]. George et al. performed an Ames test to show that Au-NPs did not induce gene mutations [2]. Nam et al. performed an SOS chromotest and reported that Au-NPs had no genotoxic potential [13]. The negative results obtained in bacterial assays might be due to the inability of Au-NPs to penetrate the bacterial cell wall. In addition, one group used LC/MS/MS analysis to measure the oxidatively modified DNA lesions in HepG2 cells treated with Au-NPs (10, 30 or $60 \mathrm{~nm}$ ) for 24 h, but the results were negative [14].

\section{In vivo studies}

Eight in vivo studies investigated the genotoxicity of Au-NPs using a comet assay, an MN assay, a random amplified polymorphic DNA-based methodology and a somatic mutation and recombination test.

\section{In vivo comet assay}

Three studies have been conducted using the in vivo comet assay and, of these, two indicated positive results. Rats received a single intraperitoneal injection (acute) or a daily single intraperitoneal injection of Au-NPs for 28 days (chronic). The cerebral cortices were isolated for the evaluation of DNA damage by the comet assay. The data showed that the acute and chronic administration of Au-NPs led to higher levels of DNA damage in the cerebral cortex compared with the negative control [15]. Fish were exposed to Au-NPs (4, 80 and $1600 \mu \mathrm{g} / \mathrm{l})$ for $96 \mathrm{~h}$ and DNA damage in blood cells from the posterior cardinal vein, as evaluated by a comet assay, was significantly increased in the Au-NP-treated group compared with the control group [16]. In the study by Schulz et al., rats were administered Au-NPs by a single intratracheal instillation in the lung for $72 \mathrm{~h}$, after which alveolar lung cells were isolated and subjected to a comet assay. Their results showed that DNA damage was not statistically significant between the Au-NP-treated group and the control group [17].

\section{In vivo MN assay}

Four studies investigated the genotoxicity of Au-NPs using an in vivo MN assay. Xia et al. reported that Au-NPs showed non-genotoxic potential, according to an in vivo $\mathrm{MN}$ test when mice were injected intravenously with $\mathrm{Au}-\mathrm{NPs}$ once daily for 4 continuous days, but Au-NPs significantly increased the frequency of MN formation when mice were injected intravenously with Au-NPs once daily for 14 continuous days [10]. This finding suggests that the genotoxic effects of Au-NPs depend on the exposure duration, which may be because prolonged exposure time may enhance the accumulation of Au-NPs, which in turn may increase the probability of a positive response. Balansky et al. reported that a single intraperitoneal injection of Au-NPs ( 40 or $100 \mathrm{~nm} ; 3.3 \mathrm{mg} / \mathrm{kg}$ bodyweight) into adult male and female Swiss mice did not induce an obvious increase in the frequency of MN formation. However, the frequency of MN formation was significantly increased in the peripheral blood and liver of mouse fetuses in the group that pregnant mice were intraperitoneally treated with $100-\mathrm{nm}$ Au-NPs (3.3 mg/kg bodyweight) on days 10,12, 14 and 17 of gestation compared with the negative control group [18]. This finding suggests that the transplacental MN assay in the liver and peripheral blood of mouse fetuses is more sensitive than the traditional $\mathrm{MN}$ assay in an adult mouse bone marrow. Two additional studies had negative MN test results for rat bone marrow cells $[17,19]$.

\section{Other in vivo studies}

Zebrafish were exposed to contaminated sediment containing Au-NPs for 20 days. The results of the random amplified polymorphic DNA assay emphasized the genotoxicity of Au-NPs [20]. Avalos et al. investigated the genotoxic potential of Au-NPs using somatic mutation and recombination test in Drosophila and the results were negative $[7]$.

\section{Conclusion}

In summary, most studies on the genotoxicity of Au-NPs have reported positive results, but several studies have reported negative responses. The inconsistency among different studies may be due to the following: different characteristics of tested materials, including particle size and surface coating; different concentrations of Au-NPs; 
different cell models; different test procedures; different genotoxicity end points; and different durations of exposure. The comet assay is very simple and cost efficient, and is relatively sensitive for screening the genotoxicity of Au-NPs in in vitro and in vivo test systems. The findings suggest that the comet assay is preferentially applied to assess the potential genotoxicity of various types of Au-NPs. The CA and gene mutation assays, which involve bacteria and usually generate negative results, may not be suitable for screening the genotoxicity of Au-NPs. In addition, although in vitro and in vivo methods are useful for assessing the genotoxicity of Au-NPs, population-based epidemiological studies on the genotoxic potential of Au-NPs are lacking. Therefore, in the future, epidemiological studies should be performed to evaluate the genotoxicity of Au-NPs among particular populations exposed to Au-NPs, which may provide direct evidence of the association between Au-NP exposure and their genotoxic potential.

Acknowledgments

We would like to thank OT Bonney for his kind help in editing the English language of this manuscript.

Financial \& competing interests disclosure

This work was funded by the National Natural Science Foundation of China (grant no. 81973105 and U1404815). The funder had no role in the preparation of the manuscript or the decision to publish. The authors have no other relevant affiliations or financial involvement with any organization or entity with a financial interest in or financial conflict with the subject matter or materials discussed in the manuscript apart from those disclosed.

OT Bonney helped in editing the English language of this manuscript.

\section{References}

1. Di Bucchianico S, Fabbrizi MR, Cirillo $S$ et al. Aneuploidogenic effects and DNA oxidation induced in vitro by differently sized gold nanoparticles. Int. J. Nanomed. 9, 2191-2204 (2014).

2. George JM, Magogotya M, Vetten MA, Buys AV, Gulumian M. From the cover: an investigation of the genotoxicity and interference of gold nanoparticles in commonly used in vitro mutagenicity and genotoxicity assays. Toxicol. Sci. 156(1), 149-166 (2017).

3. Ng CT, Li JJ, Gurung RL et al. Toxicological profile of small airway epithelial cells exposed to gold nanoparticles. Exp. Biol. Med. 238(12), 1355-1361 (2013).

4. Li JJ, Lo SL, Ng CT et al. Genomic instability of gold nanoparticle treated human lung fibroblast cells. Biomaterials 32(23), 5515-5523 (2011).

5. Di Guglielmo C, De Lapuente J, Porredon C, Ramos-Lopez D, Sendra J, Borras M. In vitro safety toxicology data for evaluation of gold nanoparticles-chronic cytotoxicity, genotoxicity and uptake. J. Nanosci. Nanotechnol. 12(8), 6185-6191 (2012).

6. Lopez-Chaves C, Soto-Alvaredo J, Montes-Bayon M, Bettmer J, Llopis J, Sanchez-Gonzalez C. Gold nanoparticles: distribution, bioaccumulation and toxicity. In vitro and in vivo studies. Nanomedicine 14(1), 1-12 (2018).

7. Avalos A, Haza AI, Mateo D, Morales P. In vitro and in vivo genotoxicity assessment of gold nanoparticles of different sizes by comet and SMART assays. Food Chem. Toxicol. 120, 81-88 (2018).

8. May S, Hirsch C, Rippl A et al. Transient DNA damage following exposure to gold nanoparticles. Nanoscale 10(33), 15723-15735 (2018).

9. Lebedova J, Hedberg YS, Odnevall Wallinder I, Karlsson HL. Size-dependent genotoxicity of silver, gold and platinum nanoparticles studied using the mini-gel comet assay and micronucleus scoring with flow cytometry. Mutagenesis 33(1), 77-85 (2018).

10. Xia Q, Li H, Liu Y, Zhang S, Feng Q, Xiao K. The effect of particle size on the genotoxicity of gold nanoparticles. J. Biomed. Mater. Res. 105(3), 710-719 (2017).

11. Paino IM, Marangoni VS, de Oliveira Rde C, Antunes LM, Zucolotto V. Cyto and genotoxicity of gold nanoparticles in human hepatocellular carcinoma and peripheral blood mononuclear cells. Toxicol. Lett. 215(2), 119-125 (2012).

12. Fraga S, Faria H, Soares ME et al. Influence of the surface coating on the cytotoxicity, genotoxicity and uptake of gold nanoparticles in human HepG2 cells. J. Appl. Toxicol. 33(10), 1111-1119 (2013).

13. Nam SH, Kim SW, An YJ. No evidence of the genotoxic potential of gold, silver, zinc oxide and titanium dioxide nanoparticles in the SOS chromotest. J. Appl. Toxicol. 33(10), 1061-1069 (2013).

14. Nelson BC, Petersen EJ, Marquis BJ et al. NIST gold nanoparticle reference materials do not induce oxidative DNA damage. Nanotoxicology 7(1), 21-29 (2013).

15. Cardoso E, Rezin GT, Zanoni ET et al. Acute and chronic administration of gold nanoparticles cause DNA damage in the cerebral cortex of adult rats. Mutat. Res. 766-767, 25-30 (2014).

16. Barreto A, Luis LG, Pinto E et al. Genotoxicity of gold nanoparticles in the gilthead seabream (Sparus aurata) after single exposure and combined with the pharmaceutical gemfibrozil. Chemosphere 220, 11-19 (2019). 
17. Schulz M, Ma-Hock L, Brill S et al. Investigation on the genotoxicity of different sizes of gold nanoparticles administered to the lungs of rats. Mutat. Res. 745(1-2), 51-57 (2012).

18. Balansky R, Longobardi M, Ganchev G et al. Transplacental clastogenic and epigenetic effects of gold nanoparticles in mice. Mutat. Res. 751-752, 42-48 (2013).

19. Jumagazieva DS, Maslyakova GN, Suleymanova LV et al. Mutagenic effect of gold nanoparticles in the micronucleus assay. Exp. Biol. Med. 151(6), 731-733 (2011).

20. Dedeh A, Ciutat A, Treguer-Delapierre M, Bourdineaud JP. Impact of gold nanoparticles on zebrafish exposed to a spiked sediment. Nanotoxicology 9(1), 71-80 (2015). 